\title{
Moment of the ratio and approximate MLEs of parameters in a bivariate Pareto distribution ${ }^{\dagger}$
}

\author{
Jungdae $\mathrm{Kim}^{1}$ \\ ${ }^{1}$ Department of Computer Information, Andong Science College \\ Received 27 August 2012, revised 21 September 2012, accepted 22 October 2012
}

\begin{abstract}
We shall derive the moment of the ratio $Y /(X+Y)$ and the reliability $P(X<Y)$, and then observe the skewness of the ratio in a bivariate Pareto density function of $(X, Y)$. And we shall consider an approximate MLE of parameters in the bivariate Pareto density function.
\end{abstract}

Keywords: Approximate maximum likelihood estimation, bivariate Pareto distribution, generalized hypergeometric function, reliability, skewness.

\section{Introduction}

Many authors have studied estimations and characterizations in a bivariate Pareto distribution with three parameters $\alpha, \beta$ and $\sigma$, whose distribution was used widely in economic applications in Johnson et al. (1994).

For two random variables $X$ and $Y$, and a real number $c$, the probability $P(X<c Y)$ is a distribution function of the ratio $Y /(X+Y)$ when $c=t /(1-t)$ for $0<t<1$.

For given random variables $X$ and $Y$, the distribution of the ratio $R=Y /(X+Y)$ is of interest in biological and physical sciences, econometrics, engineering. For example, ratios of normal variables appear as sampling distributions of single equation models in simultaneous equations models. Other area of applications includes the mass to energy ratios in nuclear physics.

The problem of estimating the probability that a random variable $X$ is less than another random variable $Y$ arises in many practical situations, like economics study. The problem has been studied by many authors for different distributions of $X$ and $Y$; see, for example Pal et al. (2005), Raqab et al. (2007) and Ali et al. (2010). Woo (2007) also studied the reliability in two independent half-triangle distributions. Moon and Lee (2009) studied an inference on the reliability $P(Y<X)$ in the Gamma case. Moon et al. (2009) studied inferences for the reliability and the ratio in an exponentiated complementary power function distribution.

$\dagger$ This research was supported by Education Capacity Enhancement Project(ECEP) Research Grants, Andong Science College in 2012.

1 Professor, Department of Computer Information, Andong Science College, Andong 760-300, Korea. E-mail: jdkim@asc.ac.kr 
Lee and Lee (2010) studied the inference on the reliability and the ratio in a right truncated Rayleigh distribution. Ali et al. (2010) studied estimations of $P(Y<X)$ when $X$ and $Y$ belong to different distribution families.

Xekalaki and Dimaki (2004) considered characterizations of a bivariate Pareto distribution. Chacko and Thomas (2007) studied the estimation of a parameter in a bivariate Pareto distribution by ranked set sampling.

In this paper, we derive the moment of the ratio $R=Y /(X+Y)$ and the reliability $P(X<Y)$, and then observe the skewness of the ratio $R=Y /(X+Y)$ in a bivariate Pareto density function of $(X, Y)$. And we consider an approximate MLE of parameters in a bivariate Pareto density function.

\section{Moment of the ratio}

A bivariate Pareto density function of $(X, Y)$ is given in Chacko and Thomas (2007) as :

$$
f(x, y)=\frac{\alpha(\alpha+1)}{\beta \sigma}\left(\frac{x}{\beta}+\frac{y}{\sigma}\right)^{-\alpha-2} \quad \text { for } x \geq \beta>0, y \geq \sigma>0,
$$

where $\alpha>0$.

From the density function (2.1), the following results can be easily obtained.

Lemma 2.1 Let $(X, Y)$ have the bivariate Pareto density function (2.1). Then (a) the marginal densities of $X$ and $Y$ are Pareto density functions with parameters $(\alpha, \beta)$ and $(\alpha, \sigma)$ respectively given by

$$
f_{X}(x)=\alpha \beta^{\alpha} x^{-\alpha-1}, \text { for } x \geq \beta
$$

and

$$
f_{Y}(y)=\alpha \sigma^{\alpha} y^{-\alpha-1}, \text { for } x \geq \sigma
$$

(b) let $W=X / Y$, the density of $W$ is given by :

$$
f_{W}(w)= \begin{cases}(\beta / \sigma)^{\alpha+1}\left[(w+\beta / \sigma)^{-2} w^{-\alpha}+\alpha(w+\beta / \sigma)^{-1} w^{-\alpha-1}\right], & \text { if } w \geq \beta / \sigma \\ (\sigma / \beta)^{\alpha-1}\left[(w+\beta / \sigma)^{-2} w^{\alpha}+\alpha \sigma / \beta(w+\beta / \sigma)^{-1} w^{\alpha-1}\right], & \text { if } 0<w \leq \beta / \sigma .\end{cases}
$$

Remark 2.2 We can easily show that $\int_{0}^{\infty} f_{W}(w) d w=1$ from formula 2.7 in Oberhettinger (1974).

From the bivariate Pareto density function (2.1), formula 2.22 in Oberhettinger (1974) and formula 7.512(5) in Gradshteyn and Ryzhik (1965), we can obtain the following results:

Proposition 2.3 Let $(X, Y)$ have the bivariate Pareto density function (2.1). Then,

$$
E\left(X^{k} \cdot Y^{m}\right)=\frac{\alpha(\alpha+1)}{(\alpha+1-k)(\alpha+1-m)} \cdot{ }_{3} F_{2}(1, \alpha+2, \alpha+1-m ; \alpha+2-k, \alpha+2-m ; 1) \cdot \beta^{k} \sigma^{m}
$$


provided that $\alpha+1>k$ and $\alpha+1>m$, where ${ }_{3} F_{2}(a, b, c ; d ; e ; x)$ is the generalized hypergeometric function.

\section{Proof :}

$$
\begin{aligned}
E\left(X^{k} Y^{m}\right) & =\frac{\alpha(\alpha+1)}{\beta \sigma} \int_{\sigma}^{\infty} \int_{\beta}^{\infty} x^{k} y^{m}(x / \beta+y / \sigma)^{-\alpha-2} d x d y \\
& =\frac{\alpha(\alpha+1)}{\sigma} \beta^{k} \int_{\sigma}^{\infty} y^{m} \int_{1}^{\infty} t^{k}(t+y / \sigma)^{-\alpha-2} d t d y \\
& =\frac{\alpha(\alpha+1)}{\alpha+1-k} \beta^{k} \sigma^{\alpha+1} \int_{\sigma}^{\infty} y_{2}^{m-\alpha-2} F_{1}(1, \alpha+2 ; \alpha+2-k ; \sigma / y) d y \\
& =\frac{\alpha(\alpha+1)}{\alpha+1-k} \beta^{k} \sigma^{m} \int_{0}^{1} t_{2}^{m-\alpha} F_{1}(1, \alpha+2 ; \alpha+2-k ; t) d t, \text { by setting } t=\sigma / y \\
& =\frac{\alpha(\alpha+1)}{(\alpha+1-k)(\alpha+1-m)} \cdot{ }_{3} F_{2}(1, \alpha+2, \alpha+1-m ; \alpha+2-k, \alpha+2-m ; 1) \cdot \beta^{k} \sigma^{m} .
\end{aligned}
$$

This completes the proof.

Especially, if $k=m=1$, then

$$
E(X Y)=[1+1 /(\alpha-1)+1 /(\alpha-2)] \beta \sigma .
$$

From Lemma 2.1(a) and the result (2.2), we can obtain the correlation coefficient between $X$ and $Y$ :

$$
\rho_{X, Y}=1 / \alpha, \alpha>2 .
$$

From Lemma 2.1 (b) and formula 2.8 in Oberhettinger (1974), we can obtain the following result for reliability $P(X<Y)$.

Proposition 2.4 Let $(X, Y)$ have the bivariate Pareto density function (2.1). Then $P(X<Y)=\left\{\begin{array}{l}1-(\beta / \sigma)^{\alpha} /(1+\beta / \sigma), \text { if } \sigma>\beta \\ (\sigma / \beta)^{\alpha-1}\left[\alpha \cdot Y(-\sigma / \beta, 1, \alpha)+\alpha \sigma / \beta \cdot Y(-\sigma / \beta, 1, \alpha+1)-(1+\beta / \sigma)^{-1}\right], \text { if } \beta>\sigma,\end{array}\right.$ where $Y(z, s, a)=\sum_{i=0}^{\infty}(a+i)^{-s} \cdot z^{i}, \quad$ if $|z|<1, a \neq 0,-1,-2, \ldots$, is Lerch's zeta function (Gradshteyn and Ryzhik, 1965, 9.55).

Proof : For $\sigma>\beta$,

$$
P(X<Y)=1-P(X / Y>1)=1-(\beta / \sigma)^{\alpha} /(1+\beta / \sigma) .
$$


For $\sigma<\beta$, by using the 2nd expression in the density of $\mathrm{W}$,

$$
\begin{aligned}
P(X<Y) & =P(X / Y<1)=(\sigma / \beta)^{\alpha-1}\left[\int_{0}^{1} w^{\alpha}(w+\beta / \sigma)^{-2} d w+\alpha \frac{\sigma}{\beta} \int_{0}^{1} w^{\alpha-1}(w+\beta / \sigma)^{-1} d w\right] \\
& =(\sigma / \beta)^{\alpha-1}\left[\alpha \frac{\sigma}{\beta} \int_{0}^{1} w^{\alpha}(w+\beta / \sigma)^{-1} d w-(1+\beta / \sigma)^{-1}+\alpha \int_{0}^{1} w^{\alpha-1}(w+\beta / \sigma)^{-1} d w\right] \\
& =(\sigma / \beta)^{\alpha-1}\left[\alpha \cdot Y(-\sigma / \beta, 1, \alpha)+\alpha \sigma / \beta \cdot Y(-\sigma / \beta, 1, \alpha+1)-(1+\beta / \sigma)^{-1}\right] .
\end{aligned}
$$

This completes the proof.

Next, we will calculate the moment of the ratio $R=Y /(X+Y)$ of $X$ and $Y$ having the bivariate density function (2.1).

From Lemma 2.1 (b) and $R=1 /(1+W)$, we can obtain the density of the ratio $R$ as :

Proposition 2.5 Let $(X, Y)$ have the bivariate Pareto density function (2.1). Then the density of the ratio $R$ is :

$$
f_{R}(r)= \begin{cases}(\beta / \sigma)^{\alpha+1} & {\left[(1-r+(\beta / \sigma) r)^{-2} \cdot r^{\alpha} /(1-r)^{\alpha}\right.} \\ & \left.+\alpha(1-r+(\beta / \sigma) r)^{-1} \cdot r^{\alpha} /(1-r)^{\alpha+1}\right], \quad \text { if } 0<r<\sigma /(\beta+\sigma) \\ (\sigma / \beta)^{\alpha-1} & {\left[(1-r+(\beta / \sigma) r)^{-2} \cdot(1-r)^{\alpha} / r^{\alpha}\right.} \\ & \left.+\alpha(\sigma / \beta)(1-r+(\beta / \sigma) r)^{-1} \cdot(1-r)^{\alpha} / r^{\alpha+1}\right], \quad \text { if } \sigma /(\beta+\sigma) \leq r<1 .\end{cases}
$$

From the density of the ratio $R$ in Proposition 2.5, binomial expansions 1.112(1) \& (2) and formula 3.194(1) in Gradshteyn and Ryzhik (1965), we can obtain the $k$ th moment of the ratio $R$ as:

Proposition 2.6 Let $(X, Y)$ have the bivariate Pareto density function (2.1). Then $k$ th moment of the ratio $R$ is :

$$
\begin{aligned}
E\left(R^{k}\right) & =\left(\frac{\sigma}{\beta}\right)^{k} \sum_{i=1}^{\infty}(-1)^{i-1} \cdot \frac{(1-\sigma / \beta)^{i-1}}{(\alpha+k+i)(1+\sigma / \beta)^{\alpha+i}} \cdot\left[i \cdot{ }_{2} F_{1}(\alpha, \alpha+k+i ; \alpha+k+i+1 ; \sigma /(\beta+\sigma))\right. \\
& \left.+\alpha_{2} F_{1}(\alpha+1, \alpha+k+i ; \alpha+k+i+1 ; \sigma /(\beta+\sigma))\right] \\
& +\left(\frac{\sigma}{\beta}\right)^{\alpha+1} \sum_{i=1}^{\infty} \frac{(1-\sigma / \beta)^{i-1}}{(\alpha+i)(1+\sigma / \beta)^{\alpha+i}} \cdot\left[i \cdot{ }_{2} F_{1}(\alpha-k, \alpha+i ; \alpha+i+1 ; \beta /(\beta+\sigma))\right. \\
& +\alpha_{2} F_{1}(\alpha+1-k, \alpha+k+i ; \alpha+k+i+1 ; \beta /(\beta+\sigma)),
\end{aligned}
$$

where ${ }_{2} F_{1}(a, b ; c ; x)$ is the hypergeometric function. 
Proof : By the density of the ratio $R$ in Proposition 2.5,

$$
\begin{aligned}
& \int_{0}^{\sigma /(\beta+\sigma)} r^{k+\alpha}(1-r)^{-\alpha}\left(1+\frac{\beta-\sigma}{\sigma} \cdot r\right)^{-2} d r \\
& =\sum_{i=1}^{\infty}(-1)^{i-1} i \frac{(\beta-\sigma)^{i-1}}{\sigma^{i-1}} \int_{0}^{\sigma /(\beta+\sigma)} r^{k+\alpha+i-1}(1-r)^{-\alpha} d r \\
& =\sum_{i=1}^{\infty} \frac{(-1)^{i-1} i}{k+\alpha+i} \frac{\sigma^{k+\alpha+1}(\beta-\sigma)^{i-1}}{(\beta+\sigma)^{k+\alpha+i}}{ }_{2} F_{1}\left(\alpha, k+\alpha+i ; k+\alpha+i+1 ; \frac{\sigma}{\beta+\sigma}\right) .
\end{aligned}
$$

By the similar arguments like the above, from 1.112(1) \& (2) and formula 3.194(1) in Gradshteyn and Ryzhik (1965), we can show the followings :

$$
\begin{aligned}
& \int_{0}^{\sigma /(\beta+\sigma)} r^{k+\alpha}(1-r)^{-\alpha-1}\left(1+\frac{\beta-\sigma}{\sigma} \cdot r\right)^{-1} d r \\
&= \sum_{i=1}^{\infty} \frac{(-1)^{i-1}}{k+\alpha+i} \cdot \frac{\sigma^{k+\alpha+1}(\beta-\sigma)^{i-1}}{(\beta+\sigma)^{k+\alpha+i}}{ }_{2} F_{1}\left(\alpha+1, k+\alpha+i ; k+\alpha+i+1 ; \frac{\sigma}{\beta+\sigma}\right), \\
& \frac{\sigma^{2}}{\beta^{2}} \int_{0}^{\beta /(\beta+\sigma)} x^{\alpha}(1-x)^{k-\alpha}\left(1-\frac{\beta-\sigma}{\beta} \cdot x\right)^{-2} d x \\
&=\frac{\sigma^{2}}{\beta^{2}} \sum_{i=1}^{\infty} \frac{i}{\alpha+i} \cdot \frac{\beta^{\alpha+1}(\beta-\sigma)^{i-1}}{(\beta+\sigma)^{\alpha+i}}{ }_{2} F_{1}\left(\alpha-k, k+\alpha+i ; k+\alpha+i+1 ; \frac{\beta}{\beta+\sigma}\right),
\end{aligned}
$$

and

$$
\begin{aligned}
\frac{\sigma}{\beta} \int_{0}^{\beta /(\beta+\sigma)} & x^{\alpha}(1-x)^{k-\alpha-1}\left(1-\frac{\beta-\sigma}{\beta} \cdot x\right)^{-1} d x \\
& =\frac{\sigma}{\beta} \sum_{i=1}^{\infty} \frac{1}{\alpha+i} \cdot \frac{\beta^{\alpha+1}(\beta-\sigma)^{i-1}}{(\beta+\sigma)^{\alpha+i}}{ }_{2} F_{1}\left(\alpha-k+1, k+\alpha+i ; k+\alpha+i+1 ; \frac{\beta}{\beta+\sigma}\right) .
\end{aligned}
$$

This completes the proof.

From Proposition 2.6 and recursion formulas of the hypergeometric function in Abramowitz and Stegun (1970), we can calculate approximate means, variances and coefficients of the skewness of the density of the ratio $\mathrm{R}$ given in Table 2.1 .

From Table 2.1, we observe the following trends for the density of the ratio $R$.

Fact 2.7 Let $(X, Y)$ have a bivariate Pareto density function (2.1) with parameters $(\alpha, \beta, \sigma)$. Then for $\alpha=0.5$ and 2 , the density $f_{R}(r)$ of the ratio $R=Y /(X+Y)$ is right skewed when $\sigma<\beta$, but it's left skewed when $\sigma>\beta$. 
Table 2.1 Approximate means, variances and coefficients of the skewness of the density function of the ratio $R$

\begin{tabular}{|c|c|c|c|c|c|c|c|}
\hline \multirow{2}{*}{$\beta$} & \multirow{2}{*}{$\sigma$} & \multicolumn{3}{|c|}{$\alpha=0.5$} & \multicolumn{3}{|c|}{$\alpha=2$} \\
\hline & & mean & variance & skewness & mean & variance & skewness \\
\hline \multirow{5}{*}{$1 / 4$} & $1 / 4$ & .5 & .04793 & 0 & .5 & .01480 & 0 \\
\hline & $1 / 2$ & .63671 & .04346 & -0.63761 & .65767 & .01255 & -0.70640 \\
\hline & 1 & .75539 & .03262 & -1.32771 & .78757 & .00778 & -1.45930 \\
\hline & 2 & .84608 & .02067 & -2.14380 & .87818 & .00371 & -2.31245 \\
\hline & 4 & .90485 & .01136 & -3.19362 & .93392 & .00144 & -3.31162 \\
\hline \multirow{5}{*}{$1 / 2$} & $1 / 4$ & .36329 & .04346 & 0.63761 & .34233 & .01255 & 0.70640 \\
\hline & $1 / 2$ & .5 & .04793 & 0 & .5 & .01480 & 0 \\
\hline & 1 & .63671 & .04346 & -0.63761 & .65767 & .01255 & -0.70640 \\
\hline & 2 & .75539 & .03262 & -1.32771 & .78757 & .00778 & -1.45930 \\
\hline & 4 & .84608 & .02067 & -2.14380 & .87818 & .00371 & -2.31245 \\
\hline \multirow{5}{*}{1} & $1 / 4$ & .24461 & .03262 & 1.32771 & .21243 & .00778 & 1.45930 \\
\hline & $1 / 2$ & .36329 & .04346 & 0.63761 & .34233 & .01255 & 0.70640 \\
\hline & 1 & .5 & .04793 & 0 & .5 & .01480 & 0 \\
\hline & 2 & .63671 & .04346 & -0.63761 & .65767 & .01255 & -0.70640 \\
\hline & 4 & .75539 & .03262 & -1.32771 & .78757 & .00778 & -1.45930 \\
\hline \multirow{5}{*}{2} & $1 / 4$ & .15392 & .02067 & 2.14380 & .12182 & .00371 & 2.31245 \\
\hline & $1 / 2$ & .24461 & .03262 & 1.32771 & .21243 & .00778 & 1.45930 \\
\hline & 1 & .36329 & .04346 & 0.63761 & .34233 & .01255 & 0.70640 \\
\hline & 2 & .5 & .04793 & 0 & .5 & .01480 & 0 \\
\hline & 4 & .63671 & .04346 & -0.63761 & .65767 & .01255 & -0.70640 \\
\hline \multirow{5}{*}{4} & $1 / 4$ & .09155 & .01136 & 3.19362 & .06608 & .00144 & 3.31162 \\
\hline & $1 / 2$ & .15392 & .02067 & 2.14380 & .12182 & .00371 & 2.31245 \\
\hline & 1 & .24461 & .03262 & 1.32771 & .21243 & .00144 & 1.45930 \\
\hline & 2 & .36329 & .04346 & 0.63761 & .34233 & .01255 & 0.70640 \\
\hline & 4 & .5 & .04793 & 0 & .5 & .01480 & 0 \\
\hline
\end{tabular}

\section{Approximate MLEs of parameters}

The following another type of a bivariate Pareto density is as given in Arnold (1983, Chapter 3) as :

$$
f(x, y)=\alpha(\alpha+1) \theta_{1}^{\alpha+1} \theta_{2}^{\alpha+1}\left(\theta_{2} x+\theta_{1} y+\theta_{1} \theta_{2}\right), \text { for } x>0 \text { and } y>0,
$$

where $\alpha>0$ and $\theta_{i}>0$ for $i=1,2$.

The marginal density functions of $X$ and $Y$ are given by :

$$
f_{X}(x)=\alpha \theta_{1}^{\alpha}\left(x+\theta_{1}\right)^{-\alpha-1}, \text { for } x>0
$$

and

$$
f_{Y}(y)=\alpha \theta_{2}^{\alpha}\left(y+\theta_{2}\right)^{-\alpha-1}, \text { for } y>0
$$

And means, variances for $X$ and $Y$ and covariance between $X$ and $Y$ are given by : 
when $\alpha>2$,

and

$$
\begin{aligned}
& E(X)=\frac{\theta_{1}}{\alpha-1}, \quad \operatorname{Var}(X)=\frac{\alpha \theta_{1}^{2}}{(\alpha-1)^{2}(\alpha-2)}, \\
& E(Y)=\frac{\theta_{2}}{\alpha-1}, \quad \operatorname{Var}(Y)=\frac{\alpha \theta_{2}^{2}}{(\alpha-1)^{2}(\alpha-2)},
\end{aligned}
$$

$$
\eta=\operatorname{Cov}(X, Y)=\frac{\theta_{1} \theta_{2}}{(\alpha-1)(\alpha-2)}-\frac{\theta_{1} \theta_{2}}{(\alpha-1)^{2}} .
$$

Let $\left(X_{1}, Y_{1}\right), \ldots,\left(X_{n}, Y_{n}\right)$ be a random sample from the density (3.1). Then from moments in (3.2), moment estimates $\bar{\alpha}, \bar{\theta}_{1}, \bar{\theta}_{2}$ of $\alpha, \theta_{1}, \theta_{2}$ respectively are given by :

$$
\begin{aligned}
& \bar{\alpha}=\frac{2 \bar{x} \bar{y}-3 \overline{x y}-\sqrt{(2 \bar{x} \bar{y}-3 \overline{x y})^{2}-4(\bar{x} \bar{y}-\overline{x y}) *(\bar{x} \bar{y}-2 \overline{x y})}}{2(\bar{x} \bar{y}-\overline{x y})}, \\
& \bar{\theta}_{1}=\bar{x}(\bar{\alpha}-1),
\end{aligned}
$$

and

$$
\bar{\theta}_{2}=\bar{y}(\bar{\alpha}-1),
$$

where $\bar{x}=(1 / n) \sum_{i=1}^{n} x_{i}, \bar{y}=(1 / n) \sum_{i=1}^{n} y_{i}$ and $\overline{x y}=(1 / n) \sum_{i=1}^{n} x_{i} y_{i}$.

Now, we consider the likelihood function to derive MLEs of $\alpha, \theta_{1}$ and $\theta_{2}$ in density function (3.1). The log-likelihood function $l\left(\alpha, \theta_{1}, \theta_{2}\right)$ of $\alpha, \theta_{1}$ and $\theta_{2}$ in density (3.1) is given by :

$$
\begin{aligned}
l\left(\alpha, \theta_{1}, \theta_{2}\right)= & n \ln \alpha+n \ln (1+\alpha)+n(\alpha+1) \ln \theta_{1}+n(\alpha+1) \ln \theta_{2} \\
& -(\alpha+2) \sum_{i=1}^{n} \ln \left(\theta_{2} x_{i}+\theta_{1} y_{i}+\theta_{1} \theta_{2}\right) .
\end{aligned}
$$

We can obtain the MLEs of $\alpha, \theta_{1}$ and $\theta_{2}$ by solving the following equations.

$$
\begin{aligned}
& 0=\frac{\partial l}{\partial \theta_{1}} \equiv p\left(\alpha, \theta_{1}, \theta_{2}\right)=n(\alpha+1) \frac{1}{\theta_{1}}-(\alpha+2) \sum_{i=1}^{n} \frac{y_{i}+\theta_{2}}{\theta_{2} x_{i}+\theta_{1} y_{i}+\theta_{1} \theta_{2}}, \\
& 0=\frac{\partial l}{\partial \theta_{2}} \equiv q\left(\alpha, \theta_{1}, \theta_{2}\right)=n(\alpha+1) \frac{1}{\theta_{2}}-(\alpha+2) \sum_{i=1}^{n} \frac{x_{i}+\theta_{1}}{\theta_{2} x_{i}+\theta_{1} y_{i}+\theta_{1} \theta_{2}},
\end{aligned}
$$

and

$$
0=\frac{\partial l}{\partial \alpha} \equiv r\left(\alpha, \theta_{1}, \theta_{2}\right)=\frac{n}{\alpha}+\frac{n}{1+\alpha}+n \ln \left(\theta_{1} \theta_{2}\right)-\sum_{i=1}^{n} \ln \left(\theta_{2} x_{i}+\theta_{1} y_{i}+\theta_{1} \theta_{2}\right) .
$$

Since approximate MLE usually performs better than the moment estimator in the sense of MSE in Balakrishnan and Cohen (1991) and Son and Woo (2009), approximate MLE could be useful in a parametric estimation only when MLE can not be represented by closed form. From equations in (3.4), since MLEs $\widehat{\alpha}, \widehat{\theta}_{1}$ and $\widehat{\theta}_{2}$ can not explicitly be represented by closed form, we consider an approximate MLEs $\widehat{\alpha}, \widehat{\theta}_{1}$ and $\widehat{\theta}_{2}$ of $\alpha, \theta_{1}$ and $\theta_{2}$ respectively. 
Based on method of finding approximate MLE of parameter in a distribution in Balakrishnan and Cohen (1991), from equations (3.4), as taking first two terms of Taylor's series for $p\left(\alpha, \theta_{1}, \theta_{2}\right), q\left(\alpha, \theta_{1}, \theta_{2}\right)$ and $r\left(\alpha, \theta_{1}, \theta_{2}\right)$ about moment estimates $\left(\bar{\alpha}, \bar{\theta}_{1}, \bar{\theta}_{2}\right)$ in $(3.3)$, an approximate MLEs $\widehat{\alpha}, \widehat{\theta}_{1}$ and $\widehat{\theta}_{2}$ of $\alpha, \theta_{1}$ and $\theta_{2}$ are obtained by the following process :

$$
\text { Setting } p_{0} \equiv p\left(\bar{\alpha}, \bar{\theta}_{1}, \bar{\theta}_{2}\right), q_{0} \equiv q\left(\bar{\alpha}, \bar{\theta}_{1}, \bar{\theta}_{2}\right) \text { and } r_{0} \equiv r\left(\bar{\alpha}, \bar{\theta}_{1}, \bar{\theta}_{2}\right) \text {. }
$$

Let $p_{\alpha} \equiv p_{\alpha}\left(\bar{\alpha}, \bar{\theta}_{1}, \bar{\theta}_{2}\right), p_{\theta_{1}} \equiv p_{\theta_{1}}\left(\bar{\alpha}, \bar{\theta}_{1}, \bar{\theta}_{2}\right)$ and $p_{\theta_{2}} \equiv p_{\theta_{2}}\left(\bar{\alpha}, \bar{\theta}_{1}, \bar{\theta}_{2}\right)$ for partial derivative of $p\left(\alpha, \theta_{1}, \theta_{2}\right)$ with respect to $\alpha, \theta_{1}$, and $\theta_{2}$, respectively. Then

$$
\begin{aligned}
& p_{\alpha=\frac{n}{\bar{\theta}_{1}}}-\sum_{i=1}^{n} \frac{y_{i}+\bar{\theta}_{2}}{\bar{\theta}_{2} x_{i}+\bar{\theta}_{1} y_{i}+\bar{\theta}_{1} \cdot \bar{\theta}_{2}}, \\
& p_{\theta_{1}}=-n(\bar{\alpha}+1) \frac{1}{\left(\bar{\theta}_{1}\right)^{2}}+(\bar{\alpha}+2) \sum_{i=1}^{n} \frac{\left(y_{i}+\bar{\theta}_{2}\right)^{2}}{\left(\bar{\theta}_{2} x_{i}+\bar{\theta}_{1} y_{i}+\bar{\theta}_{1} \cdot \bar{\theta}_{2}\right)^{2}},
\end{aligned}
$$

and

$$
p_{\theta_{2}}=(\bar{\alpha}+2) \sum_{i=1}^{n} \frac{x_{i} y_{i}}{\left(\bar{\theta}_{2} x_{i}+\bar{\theta}_{1} y_{i}+\bar{\theta}_{1} \cdot \bar{\theta}_{2}\right)^{2}} .
$$

Let $q_{\alpha} \equiv q_{\alpha}\left(\bar{\alpha}, \bar{\theta}_{1}, \bar{\theta}_{2}\right), q_{\theta_{1}} \equiv q_{\theta_{1}}\left(\bar{\alpha}, \bar{\theta}_{1}, \bar{\theta}_{2}\right)$ and $q_{\theta_{2}} \equiv q_{\theta_{2}}\left(\bar{\alpha}, \bar{\theta}_{1}, \bar{\theta}_{2}\right)$ for partial derivatives of $q\left(\alpha, \theta_{1}, \theta_{2}\right)$ with respect to $\alpha, \theta_{1}$, and $\theta_{2}$, respectively. Then

$$
\begin{aligned}
& q_{\alpha}=\frac{n}{\bar{\theta}_{2}}-\sum_{i=1}^{n} \frac{x_{i}+\bar{\theta}_{1}}{\bar{\theta}_{2} x_{i}+\bar{\theta}_{1} y_{i}+\bar{\theta}_{1} \cdot \bar{\theta}_{2}}, \\
& q_{\theta_{1}}=(\bar{\alpha}+2) \sum_{i=1}^{n} \frac{x_{i} y_{i}}{\left(\bar{\theta}_{2} x_{i}+\bar{\theta}_{1} y_{i}+\bar{\theta}_{1} \cdot \bar{\theta}_{2}\right)^{2}},
\end{aligned}
$$

and

$$
q_{\theta_{2}}=-n(\bar{\alpha}+1) \frac{1}{\left(\bar{\theta}_{2}\right)^{2}}+(\bar{\alpha}+2) \sum_{i=1}^{n} \frac{\left(x_{i}+\bar{\theta}_{1}\right)^{2}}{\left(\bar{\theta}_{2} x_{i}+\bar{\theta}_{1} y_{i}+\bar{\theta}_{1} \bar{\theta}_{2}\right)^{2}}
$$

And let $r_{\alpha} \equiv r_{\alpha}\left(\bar{\alpha}, \bar{\theta}_{1}, \bar{\theta}_{2}\right), r_{\theta_{1}} \equiv r_{\theta_{1}}\left(\bar{\alpha}, \bar{\theta}_{1}, \bar{\theta}_{2}\right)$ and $r_{\theta_{2}} \equiv r_{\theta_{2}}\left(\bar{\alpha}, \bar{\theta}_{1}, \bar{\theta}_{2}\right)$ for partial derivatives of $r\left(\alpha, \theta_{1}, \theta_{2}\right)$ with respect to $\alpha, \theta_{1}$, and $\theta_{2}$, respectively. Then

$$
\begin{aligned}
& r_{\alpha}=-\frac{n}{(\bar{\alpha})^{2}}-\frac{n}{(1+\bar{\alpha})^{2}}, \\
& r_{\theta_{1}}=\frac{n \bar{\theta}_{2}}{\bar{\theta}_{1} \bar{\theta}_{2}}-\sum_{i=1}^{n} \frac{y_{i}+\bar{\theta}_{2}}{\left.\bar{\theta}_{2} x_{i}+\bar{\theta}_{1} y_{i}+\bar{\theta}_{1} \bar{\theta}_{2}\right)},
\end{aligned}
$$

and

$$
r_{\theta_{2}}=\frac{n \bar{\theta}_{1}}{\bar{\theta}_{1} \bar{\theta}_{2}}-\sum_{i=1}^{n} \frac{x_{i}+\bar{\theta}_{1}}{\left(\bar{\theta}_{2} x_{i}+\bar{\theta}_{1} y_{i}+\bar{\theta}_{1} \bar{\theta}_{2}\right)} .
$$


Therefore, we can obtain the following asymptotic linear equations:

and

$$
\begin{aligned}
& 0 \approx p_{0}+p_{\alpha} \cdot(\alpha-\bar{\alpha})+p_{\theta_{1}} \cdot\left(\theta_{1}-\bar{\theta}_{1}\right)+p_{\theta_{2}} \cdot\left(\theta_{2}-\bar{\theta}_{2}\right), \\
& 0 \approx q_{0}+q_{\alpha} \cdot(\alpha-\bar{\alpha})+q_{\theta_{1}} \cdot\left(\theta_{1}-\bar{\theta}_{1}\right)+q_{\theta_{2}} \cdot\left(\theta_{2}-\bar{\theta}_{2}\right),
\end{aligned}
$$

$$
0 \approx r_{0}+r_{\alpha} \cdot(\alpha-\bar{\alpha})+r_{\theta_{1}} \cdot\left(\theta_{1}-\bar{\theta}_{1}\right)+r_{\theta_{2}} \cdot\left(\theta_{2}-\bar{\theta}_{2}\right) .
$$

From linear equations in (3.9) approximate MLEs $\widehat{\alpha}, \widehat{\theta_{1}}$ and $\widehat{\theta_{2}}$ of $\alpha, \theta_{1}$ and $\theta_{2}$, respectively are obtained as follows :

Proposition 3.1 Let $\left(X_{1}, Y_{1}\right), \cdots,\left(X_{n}, Y_{n}\right)$ be a sample from the bivariate Pareto density function (3.1). Then approximate MLEs $\widehat{\alpha}, \widehat{\theta}_{1}$ and $\widehat{\theta}_{2}$ of $\alpha, \theta_{1}$ and $\theta_{2}$ are given by :

$$
\begin{aligned}
& \widehat{\alpha} \approx \bar{\alpha}+\operatorname{det}\left(D_{1}\right) / \operatorname{det}(D), \\
& \widehat{\theta}_{1} \approx \bar{\theta}_{1}+\operatorname{det}\left(D_{2}\right) / \operatorname{det}(D),
\end{aligned}
$$

and

$$
\widehat{\theta}_{2} \approx \bar{\theta}_{2}+\operatorname{det}\left(D_{3}\right) / \operatorname{det}(D),
$$

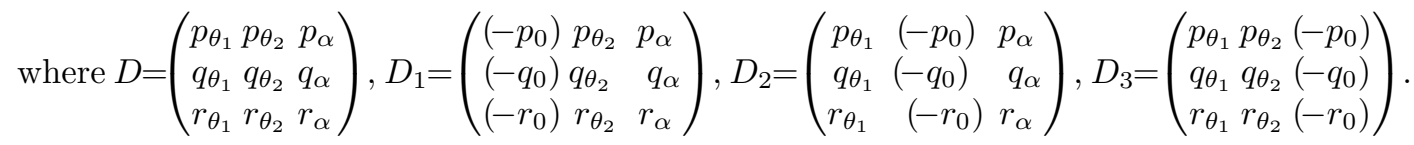

To assure the result in Proposition 3.1, the following Example provides only numerical values of approximate MLEs of parameters without doing a fit of goodness for the bivariate density function (3.1).

Example 3.1 (Neter and Wasserman, 1974)

Let $(X, Y)$ be denoted by a pair of number of dispensers, $X$, and coffee sales (hundred gallons), $Y$, which its sales are measured in hundreds of gallons of coffee sold. Assume pairs $\left(x_{i}, y_{i}\right)$ come from the bivariate density function (3.1).

\begin{tabular}{cccccccc}
$X=x$ & 0 & 0 & 1 & 1 & 2 & 2 & 4 \\
$Y=y$ & 45.1 & 48.4 & 108.2 & 117.3 & 211.7 & 217.0 & 455.3 \\
\hline$X=x$ & 4 & 5 & 5 & 6 & 6 & 7 & 7 \\
$Y=y$ & 458.9 & 586.7 & 592.1 & 741.4 & 731.8 & 1654.7 & 1871.5
\end{tabular}

From moment estimates in (2.3),

$$
\bar{a}=3.73315, \bar{\theta}_{1}=9.76124 \text {, and } \bar{\theta}_{2}=1530.56 .
$$

And from (3.4) and (3.5), $p_{0}=0.084216, q_{0}=-0.000012$, and $r_{0}=-0.208861$. From (3.6), $p_{\alpha}=-0.1269043, p_{\theta_{1}}=0.000038235$, and $p_{\theta_{2}}=0.2648561$. From (3.7), $q_{\alpha}=0.0000388235$, $q_{\theta_{1}}=4.344508 E-6$ and $q_{\theta_{2}}=0.0015745$. And from (3.8), $r_{\alpha}=0.02648561, r_{\theta_{1}}=0.0015745$, and $r_{\theta_{2}}=-1.6294874$. From Proposition 3.1, approximate MLEs $\widehat{\alpha}, \widehat{\theta}_{1}$ and $\widehat{\theta}_{2}$ of $\alpha, \theta_{1}$ and $\theta_{2}$ are given by :

$$
\widehat{\alpha} \approx 4.048, \widehat{\theta}_{1} \approx 9.511 \text { and } \widehat{\theta}_{2} \approx 1530.405
$$




\section{References}

Abramowitz, M. and Stegun, I. A. (1970). Handbook of mathematical functions, Dover Publications Inc., New York.

Ali, M. M., Pal, M. and Woo, J. (2010). Estimation of $P(Y<X)$ when $X$ and $Y$ belong to different distribution families. Journal of Probability and Statistical Science, 8, 19-33.

Arnold, B. C. (1983). Pareto distributions, International Co-operative Publishing House, Maryland.

Balakrishnan, N. and Cohen, A. C. (1991). Order statistics and inference, Academic Press, Inc., New York.

Chacko, M. and Thomas, P. Y. (2007). Estimation of a parameter of bivariate Pareto distribution by ranked set sampling. Journal of Applied Statistics, 34, 703-714.

Gradshteyn, I. S. and Ryzhik, I. M. (1965). Tables of integrals, series, and products, Academic Press, New York.

Johnson, N. L., Kotz, S. and Balakrishnan, N. (1994). Continue univariate distribution, Houghton Mifflin Co., Boston.

Moon, Y. G. and Lee, C. S. (2009). Inference on reliability $P(Y<X)$ in the gamma case. Journal of the Korean Data 8 Information Science Society, 20, 219-223.

Moon, Y. G., Lee, C. S. and Ryu, S. G. (2009). Reliability and ratio in exponentiated complementary power function distribution. Journal of the Korean Data \& Information Sciences Society, 20, 955-960.

Neter, J. and Wasserman, W. (1974). Applied linear statistical models, Richard D. Irwin Inc., Homewood, Illinois.

Lee, J. C. and Lee, C. S. (2010). Reliability and ratio in a right truncated Rayleigh distribution. Journal of the Korean Data 83 Information Science Society, 21, 195-200.

Oberhettinger, F. (1974). Tables of Mellin transforms, Springer-Verlag, New York.

Pal, M., Ali, M. M. and Woo, J. (2005). Estimation and testing of $P(Y<X)$ in two parameter exponential distributions. Statistics, 39, 415-428.

Raqab, M. Z., Madi, M. T. and Kundu, D. (2007). Estimation of $P(Y<X)$ for a 3-parameter generalized exponential distribution. Communications in Statistics-Theory and Methods, 37, 2854-2864.

Son, H. and Woo, J. (2009). Estimations in a skewed double Weibull distribution. Communications of the Korean Statistical Society, 16, 859-870.

Woo, J. (2007). Reliability in a half-triangle distribution and a skew-symmetric distribution. Journal of the Korean Data 83 Information Sciences Science Society, 18, 543-552.

Xekalaki, D. and Dimaki, C. (2004). Characterizations of bivariate Pareto Yule distribution. Communications in Statistics-Theory and Methods, 33, 3033-3042. 